\title{
Open string theory and planar algebras
}

\author{
Özgür Ceyhan ${ }^{1}$ and Matilde Marcolli ${ }^{2}$ \\ ${ }^{1}$ Korteweg-de Vries Institute for Mathematics, University of Amsterdam, PO Box 94248, \\ 1090 GE Amsterdam, The Netherlands \\ 2 Mathematics Department, California Institute of Technology, 1200 E California, Blvd.f, \\ Pasadena, CA 91125, USA \\ E-mail: o.ceyhan@uva.nl and matilde@caltech.edu
}

Received 19 March 2010, in final form 21 March 2010

Published 16 August 2010

Online at stacks.iop.org/JPhysA/43/385401

\begin{abstract}
In this paper, we show that abstract planar algebras are algebras over the topological operad of moduli spaces of stable maps with Lagrangian boundary conditions, which in the case of the projective line are described in terms of real rational functions. These moduli spaces appear naturally in the formulation of open string theory on the projective line. We also show two geometric ways to obtain planar algebras from real algebraic geometry, one based on string topology and one on Gromov-Witten theory. In particular, through the wellknown relation between planar algebras and subfactors, these results establish a connection between open string theory, real algebraic geometry and subfactors of von Neumann algebras.
\end{abstract}

PACS numbers: 02.10.-v, 11.25.Hf

Mathematics Subject Classification: 14D21, 18D50, 55P50, 14N35

(Some figures in this article are in colour only in the electronic version)

\section{Introduction}

The purpose of this paper is to show that planar algebras arise as algebras over an operad of moduli spaces of stable maps to $\mathbb{P}^{1}$ with Lagrangian boundary conditions, which can be described in terms of real algebraic curves. In particular, the results presented in this paper can be interpreted as a connection between open string theory on $\mathbb{P}^{1}$ and Jones' theory of subfactors of von Neumann algebras, using, as intermediate steps, the relation between open string theory on $\mathbb{P}^{1}$ and certain moduli spaces $R_{g}\left(\mathbb{P}^{1}, d\right)$ of maps to $\mathbb{P}^{1}$ with Lagrangian boundary conditions, combined with the main result we prove here, which relates the latter to the theory of planar algebras developed in [6]. The connection to subfactors can then be seen by invoking the result of [17] and its reformulation in terms of planar algebras of [5] and [9]. This paper outlines the main points of our construction and some applications. A longer and more detailed treatment will follow elsewhere. 
The main point involved in our description of planar algebras in terms of real algebraic geometry is an identification of (weighted) planar tangles as the combinatorial datum that encodes the components of the moduli spaces $R_{g}\left(\mathbb{P}^{1}, d\right)$ of stable $M$-maps to $\mathbb{P}^{1}$ with Lagrangian boundary conditions. The terminology $M$-maps here refer to the fact that they are realized by maximal real algebraic curves. We also describe the compositions of planar tangles and the trace map in this geometric setting. More precisely, we prove the following statement.

Theorem 1.1. Abstract planar algebras are topological operads of $R_{g}\left(\mathbb{P}^{1}, d\right)$.

In particular, by restricting to the case with $g=0$, we obtain the case of the TemperleyLieb algebras.

This paper is organized as follows: the following two sections respectively review the relation between open string theory on $\mathbb{P}^{1}$ and the moduli spaces $R_{g}\left(\mathbb{P}^{1}, d\right)$, as well as the well-known relation between subfactors and planar algebras. The main original contribution of this paper is given in sections 4 and 5. In section 4, we connect the two previous topics by proving theorem 1.1 and in section 5 we then describe two different methods, both based on real algebraic geometry, for constructing planar algebras as representations of the planar operad; the first is based on algebraic loop spaces and string topology and the other is based on a real version of Gromov-Witten theory.

\section{Real algebraic geometry and open string theory}

\subsection{Complex and real curves, and bordered Riemann surfaces}

Recall that a bordered Riemann surface of type $(g, h)$ is a Riemann surface with boundary, with $g$ handles and $h$ boundary components, oriented according to the orientation induced by the complex structure on the Riemann surface. We also denote, as in [1, 7], the complex double of a bordered Riemann surface $\Sigma$ by $\Sigma_{\mathbb{C}}$, with $\sigma$ the antiholomorphic involution on $\Sigma_{\mathbb{C}}$.

2.1.1. Maximal real curves. A maximal real curve, denoted by $M$-curve, is a smooth real algebraic curve $\left(\Sigma_{\mathbb{C}}, \sigma\right)$ with the maximal number of connected components of the real part $\Sigma_{\mathbb{R}}$ of $\sigma$. By Harnack's bound this maximal number is $g+1$ for a smooth curve of genus $g$.

The real part $\Sigma_{\mathbb{R}}$ of an $M$-curve divides $\Sigma_{\mathbb{C}}$ into two two-dimensional discs, respectively denoted by $\Sigma^{+}$and $\Sigma^{-}$, minus a set of interior (open) discs $D_{1}, \ldots, D_{g}$, having $\Sigma_{\mathbb{R}}$ as their common boundary in $\Sigma_{\mathbb{C}}$. The real structure $\sigma$ interchanges $\Sigma^{ \pm}$, and the complex orientations of $\Sigma^{ \pm}$induce two opposite orientations on $\Sigma_{\mathbb{R}}$, called its complex orientations. The quotient $\bar{\Sigma}:=\Sigma_{\mathbb{C}} / \sigma$ is isomorphic to $\Sigma^{+}$. Here $\Sigma^{+} \simeq \bar{\Sigma}$ is a bordered Riemann surface and the real algebraic curve $\left(\Sigma_{\mathbb{C}}, \sigma\right)$ is its complex double, cf [18].

\subsection{Open strings on $\mathbb{P}^{1}$ and the moduli spaces of real maps}

We briefly present here the setting of open string theory on $\mathbb{P}_{\mathbb{C}}^{1}$ with a Lagrangian submanifold $\mathbb{P}_{\mathbb{R}}^{1}$. We especially focus on the role of the moduli space of stable maps with Lagrangian boundary conditions.

2.2.1. $M$-maps and their moduli spaces. We define stable $M$-maps following a setting similar to that of [7]. 
Definition 2.1. An M-map (of degree d) is a pair $\left(\Sigma^{+}, f^{+}\right)$consisting of the following data.

(A) A bordered Riemann surface $\Sigma^{+}$(of type $\left.(0, g+1)\right)$ ) whose complex double $\left(\Sigma_{\mathbb{C}}, \sigma\right)$ is an $M$-curve of genus $g$, and with boundary components $\partial \Sigma=\partial D_{*} \cup \partial D_{1} \cup \cdots \cup \partial D_{g}$.

(B) A relative map $f^{+}:\left(\Sigma^{+}, \partial \Sigma^{+}\right) \rightarrow\left(\mathbb{P}_{\mathbb{C}}^{1}, \mathbb{P}_{\mathbb{R}}^{1}\right)$ which is the restriction of a real stable morphism $f: \Sigma_{\mathbb{C}} \rightarrow \mathbb{P}_{\mathbb{C}}^{1}$ (of degree d) with generic ramification points i.e., the singularities of $f$ are all of degree 2 .

(C) A set consisting of a critical point of $f$ for each non-empty component of $\Sigma_{\mathbb{R}}$.

An M-map is stable if it does not admit any infinitesimal automorphisms.

The moduli space $R_{g}\left(\mathbb{P}^{1}, d\right)$ is the space of isomorphism classes of stable $M$-maps. The connected components of $R_{g}\left(\mathbb{P}^{1}, d\right)$ are in fact isomorphic to the components of the principal stratum of the moduli space of stable bordered maps $\bar{M}_{0 ; g+1}\left(\mathbb{P}_{\mathbb{C}}^{1}, \mathbb{P}_{\mathbb{R}}^{1}, d\right)$ in [7]. They parameterize the non-degenerate elements in the moduli space of stable bordered maps with additional decoration given in (C).

The geometric and topological properties of the space $\bar{M}_{0 ; g+1}\left(\mathbb{P}_{\mathbb{C}}^{1}, \mathbb{P}_{\mathbb{R}}^{1}, d\right)$ and its principal stratum $R_{g}\left(\mathbb{P}^{1}, d\right)$ have been extensively studied in [11]. The construction in [11] is in fact more general; it considers an arbitrary symplectic manifold $X$ and its Lagrangian manifold $L$ as the target of stable maps.

Remark 2.2. Conditions (A) and (B) in definition 2.1 guarantee that the stable $M$-maps are stable maps of bordered surfaces in the sense of [7]. However, the essence of the main focus of this paper, the planar algebras, forces us to impose the maximality plus the non-degeneracy conditions: (1) the domain curves of $M$-maps are $M$-curves, therefore, required to be smooth. This condition also guarantees that real loci of the curves contain the maximal number of ovals. (2) The real morphisms are generic. These two conditions are necessary to obtain planar tangles as combinatorial identifiers of the connected components of the moduli spaces $R_{g}\left(\mathbb{P}^{1}, d\right)$. On the other hand, the data $(\mathrm{C})$ is an additional decoration which is needed in order to avoid rotational ambiguity in the composition of planar tangle, see section 3.1.2.

\section{Planar algebras and subfactors}

\subsection{Planar algebras}

We briefly recall here, for later use, the basic definitions and facts of the theory of planar algebras, as developed in [6]. We follow closely the short survey given in [2].

3.1.1. Planar tangles. A planar $k$-tangle $T$ consists of the unit disc $D \subset \mathbb{C}$ together with a finite collection of discs $D_{1}, D_{2}, \ldots, D_{g}$ inside $D$. The boundaries of $D$ and of each interior disc $D_{i}$ are decorated by an even number of marked points, $2 k$ points on $\partial D$ and $2 k_{i}$ points on each $\partial D_{i}$. The interior of $D \backslash \bigcup_{i} D_{i}$ also contains a collection of non-intersecting strings, which are either closed strings or have as boundary the marked points on the $\partial D \cup \partial D_{i}$. Each marked point lies on the boundary of one of these strings.

The complementary region $D \backslash\left(\{\right.$ strings $\left.\} \bigcup_{i} D_{i}\right)$ admits a checkerboard black and white coloring as well as a choice of a white region at each $D_{i}$.

3.1.2. Planar operad. Two planar tangles $T$ and $S$ can be composed whenever the number of marked points on the boundary of $S$ matches the number of marked points on the boundary of one of the interior discs $D_{j}$ of $T$. The composition $T \circ_{j} S$ is then given by gluing a rescaled 


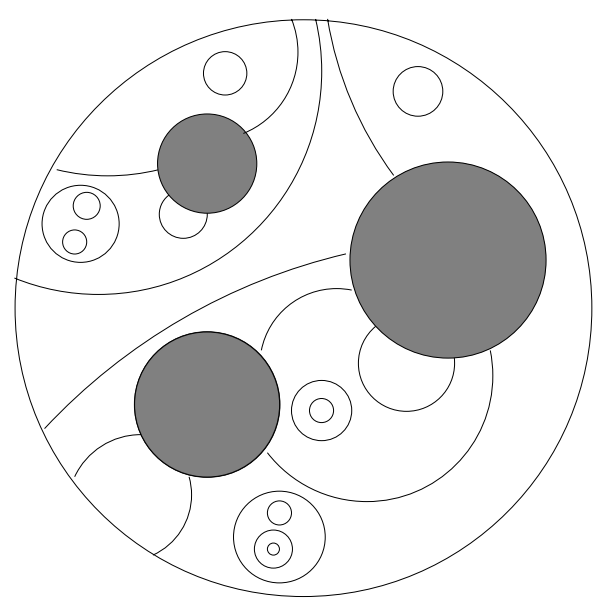

Figure 1. Planar tangle.

copy of $S$ in place of the interior of the disc $D_{j}$, so as to match the shadings and the marked white regions. This operation is well defined, with the coloring and choice of the white region eliminating any possible rotational ambiguity. Also the result only depends on the isotopy classes.

The planar operad $\mathcal{P}$ consists of all orientation-preserving diffeomorphism classes of planar $k$-tangles that fix the boundary $\partial D$. The structure of the operad is given by the compositions of tangles, defined as above. An example of compositions is given in figure 2. In figures 1 and 2, the black/white coloring and the marked white regions are not shown for simplicity.

3.1.3. Planar algebras. One then defines planar algebras $([2,6])$ as algebras over the planar operad $\mathcal{P}$, in the sense described in [12].

This means (see e.g. [2]) that a planar algebra $\mathcal{P}$ is a family of vector spaces $\left\{V_{k}\right\}_{k>0}$ together with a morphism $Z$ from the planar operad $\mathcal{P}$ to the (colored) operad Hom of multilinear maps between vector spaces.

3.1.4. Partition function and trace. The morphism $Z: \mathcal{P} \rightarrow$ Hom from the planar operad to the operad of multilinear maps of vector spaces has the following properties.

Given a tangle $T_{\ell}$, where $2 \ell$ is the number of points on $\partial D$, one obtains a multilinear map

$$
Z\left(T_{\ell}\right): \otimes_{i=1}^{n} V_{\ell_{i}} \rightarrow V_{\ell},
$$

satisfying the composition property

$$
Z\left(T \circ_{j} S\right)=Z(T) \circ_{j} Z(S) .
$$

Moreover, one has the following properties, which give $Z$ an interpretation as a 'partition function' associated with a planar algebra.

- Normalization: let $T_{0, \emptyset, b / w}$ be the colored discs of figure 3 . Then

$$
Z\left(T_{0, \emptyset, b / w}\right)=1 .
$$



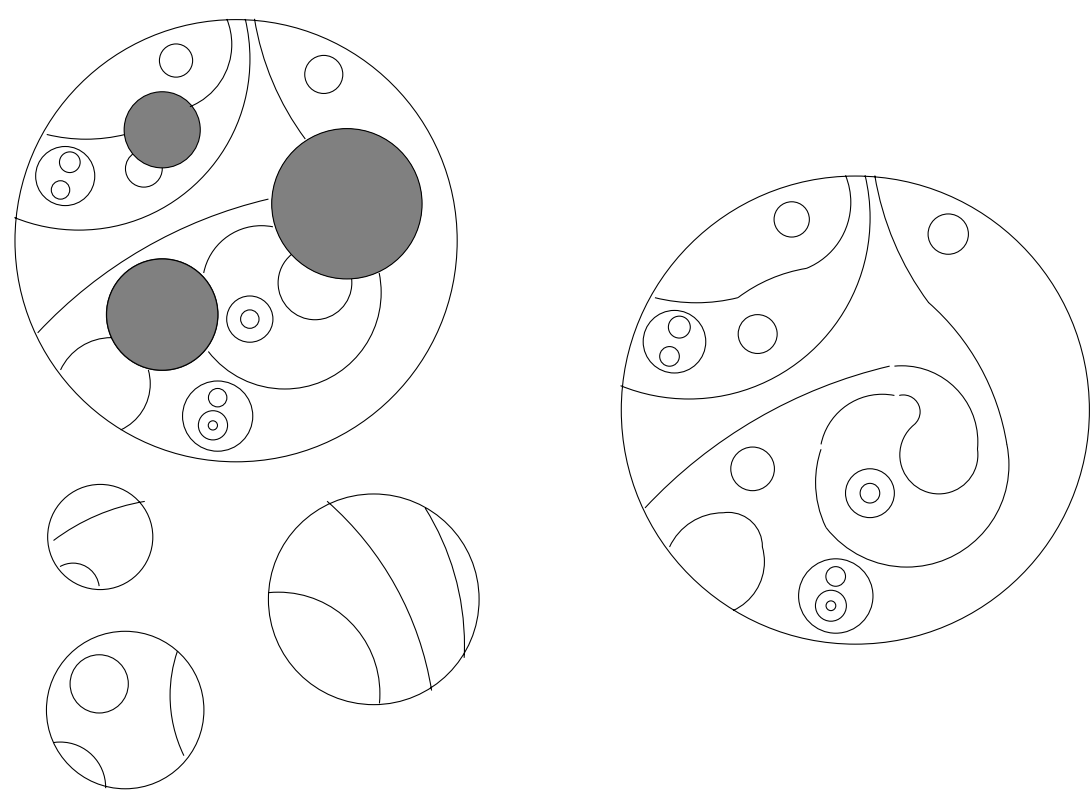

Figure 2. Planar operad: compositions of tangles.

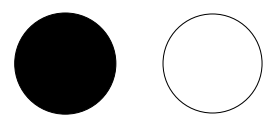

Figure 3. The planar tangles $T_{0, \emptyset, b / w}$.

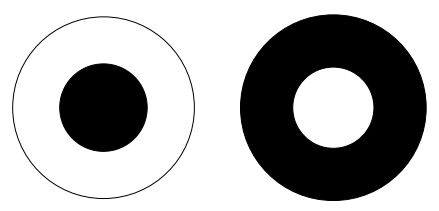

Figure 4. The planar tangles $T_{0, \emptyset, \mathcal{L}, b / w}$.

- Parameters: consider the case of planar tangles as in figure 4, given by a simple curve with no boundary inside $D$ and the two possible choices of coloring. Then the corresponding linear maps under $Z$ are of the form

$$
Z\left(T_{0, \emptyset, \mathcal{L}, b}\right)=\delta_{1}, \quad Z\left(T_{0, \emptyset, \mathcal{L}, w}\right)=\delta_{2},
$$

for two parameters $\delta_{i}$.

These two rules give a procedure to eliminate ovals from a planar tangle, replacing them in the image under $Z$ by a multiplicative factor of the form $\delta_{1}^{m} \delta_{2}^{n}$, depending on the parameters $\delta_{i}$, see figure 5 , where configurations of ovals are encoded via rooted trees and the multiplicative factor is computed accordingly.

One of the most interesting applications of planar algebras is to the theory of subfactors of von Neumann algebras [2, 5, 6, 8, 9, 17]. In [17], Popa showed that one can associate with 


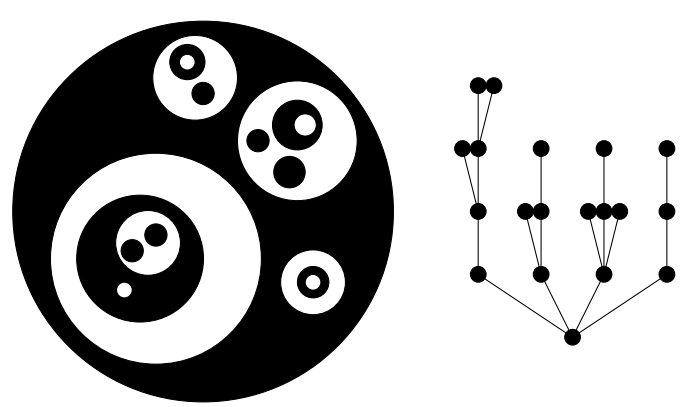

Figure 5. Ovals in planar tangles encoded by rooted trees.

a subfactor a standard invariant, which is a planar algebra. Not all planar algebras arise as the standard invariant of a subfactor, but one can characterize those that have this property, as in section 4 of [6]. We hope that relating planar algebras to the moduli spaces of real algebraic geometry may be of interest also in the subfactors perspective.

\section{Planar algebras and open string theory}

\subsection{Weighted planar tangles}

We first define weighted planar tangles, which are the planar tangles with additional decorations, and we use them to distinguish the connected components of the moduli space $R_{g}\left(\mathbb{P}^{1}, d\right)$.

Definition 4.1. Let $T$ be a planar tangle as in section 3.1.1. Then, $T$ is called weighted if each interior string and each boundary segment are decorated with a non-negative integer weight. A weighted tangle is of weight $d$ if the sum of weights for all strings and boundary segments is $d$.

Even though Jones leaves room for possible additional decorations of planar tangles, the weight data is not a part of the original definition. One can probably find a source of such decorations in geometry. For instance, in the case of planar algebra generated by tangles in 3 -spaces, framing would provide such additional data. On the other hand, there is a natural forgetful functor from weighted tangles to ordinary tangles which can be given by simply forgetting the weight data or by taking summation of all possible degrees.

\subsection{The moduli space $R_{g}\left(\mathbb{P}^{1}, d\right)$ and planar tangles}

We associate a tangle with each stable $M$-map $\left(\Sigma^{+}, f^{+}\right) \in R_{g}\left(\mathbb{P}^{1}, d\right)$ by simply pulling back the real line $\mathbb{P}_{\mathbb{R}}^{1}=\mathbb{R} \cup \infty$ by the map $f^{+}$,

$$
\Phi:\left(\Sigma^{+}, f^{+}\right) \mapsto T:=\left(f^{+}\right)^{-1}\left(\mathbb{P}_{\mathbb{R}}^{1}\right) .
$$

Let $\left(\Sigma_{\mathbb{C}}, f\right)$ be the complex double of $\left(\Sigma^{+}, f^{+}\right)$. Let Crit $(f)$ be the set of critical values of $f$, and let $\operatorname{Crit}_{\mathbb{R}}(f)$ and $\operatorname{Crit}_{\mathbb{C}}(\mathrm{f})$ denote, respectively, the set of real critical values and its complement. To determine weights, consider an arbitrary point in $x \in \mathbb{P}_{\mathbb{R}}^{1} \backslash \mathrm{Crit}_{\mathbb{R}}(f)$. For each string (or boundary segment), we define the function $w(x)$ as the number of preimages of $x$ lying on this string (or boundary segment). Then the weight of this string (or boundary 
segment) is the minimum of $w(x)$. Note that the weight of a closed string in $\Sigma^{+}$coincides with the multiplicity of $f^{+}$restricted to this string.

The following statement is a slight generalization of a similar result in [14] for genus zero covers of $\mathbb{P}^{1}$.

Proposition 4.2. The map $\Phi$ provides a 1-1 correspondence between the set of connected components of the moduli space $R_{g}\left(\mathbb{P}^{1}, d\right)$ and the set of weighted planar tangles of weight $d$.

Proof. We first check that this indeed gives a weighted planar tangle. Since $\Sigma^{+}$is half of a maximal real curve of genus $g$, it is topologically a disc minus a collection of $g$ discs. The preimage $\left(f^{+}\right)^{-1}\left(\mathbb{P}_{\mathbb{R}}^{1}\right)$ gives the strings inside $\Sigma^{+}$or segments in the boundary of $\Sigma^{+}$.

We now check the condition that these tangles are expected to satisfy. These strings intersect only in the boundary $\partial \Sigma^{+}$of $\Sigma^{+}$. We have the following possibilities.

Strings inside $\Sigma^{+}$. If the strings had intersections in $\Sigma^{+} \backslash \partial \Sigma^{+}$, then such an intersection point $z$ would be a critical point with a real critical value. Hence, the same is also true for the conjugate $\sigma(z) \in \Sigma_{\mathbb{C}}$ in the complex double $\Sigma_{\mathbb{C}}$ of $\Sigma^{+}$. However, this contradicts the genericity condition of $f: \Sigma \rightarrow \mathbb{P}_{\mathbb{C}}^{1}$ (see definition 2.1).

Strings and the boundary $\partial \Sigma^{+}$. All critical points of $f$ which have real critical values must be real. If $z$ is a critical point with a real critical value, then $\bar{z}$ is a critical point with the same critical value. Therefore, $z=\bar{z}$, since $f$ is generic. For each such critical point, $f^{-1}\left(\mathbb{P}_{\mathbb{R}}^{1}\right)$ contains exactly four arcs in $\Sigma_{\mathbb{C}}$ incident to it. Two of these arcs are the arcs lying in $\partial D_{i} \subset \mathbb{R} \Sigma$, while the other two interchange under the involution $\sigma$. In particular, the other endpoints of these two arcs coincide.

Next, we need to show that the number of critical points at each boundary component $\partial D_{i}$ of $\Sigma^{+}$is even, as required for the data to define a planar tangle. Note that the set of all critical points of the real map $f: \Sigma \rightarrow \mathbb{P}_{\mathbb{C}}^{1}$ consists of an even number of points. The order of this set is in fact $2(d+g-1)$ due to the Riemann-Hurwitz formula. On the other hand, the real maps $\left(\Sigma_{\mathbb{C}}, f\right)$ degenerate when their critical points collide, in particular when a complex conjugate pair of critical points degenerates to a real point of $\Sigma$. Under such a degeneration, it can only split into a pair of real ramification points lying in the same real component $\partial D_{i}$. Since there exist stable maps $f: \Sigma_{\mathbb{C}} \rightarrow \mathbb{P}_{\mathbb{C}}^{1}$ without any real ramification points, we show that the number of critical points is even for each boundary component $\partial D_{i}$ via a simple induction on these types of degenerations.

We need to show that, for the tangle, we associated with elements of a connected component of $R_{g}\left(\mathbb{P}^{1}, d\right)$, the isotopy class does not change. It is clear that the isotopy class of these tangles will not change under small perturbations and it can only change through intersections of strings. As we have already observed above, any intersection of strings violates the genericity condition. Namely, changes of isotopy class of tangles can happen when one passes through the discriminant locus to another component of $R_{g}\left(\mathbb{P}^{1}, d\right)$.

Finally, to show that we have a bijection, we then need to show that any arbitrary tangle of weight $d$ can be obtained in this way. For a given such tangle, an element of $R_{g}\left(P^{1}, d\right)$ can be constructed by gluing a set of weighted pairs of pants which is in fact $\Sigma^{+} \backslash\{$ strings\}. The gluing prescription of weighted pants is given in theorem 1 in [14] based on [15].

In the following, we denote by $C_{T}$ the connected component of $R_{g}\left(\mathbb{P}^{1}, d\right)$ corresponding to a given weighted tangle $T$. 
Remark 4.3. A generalization of [14] to the real meromorphic functions on separating curves has been given in [13]. They use similar tangles that are not necessarily planar to classify the connected component of the real Hurwitz spaces.

\subsection{Sewing stable M-maps}

Let $\left(\Sigma_{1}^{+}, f_{1}^{+}\right) \in C_{T_{1}}$ and $\left(\Sigma_{2}^{+}, f_{2}^{+}\right) \in C_{T_{2}}$ be a pair of stable $M$-maps such that the restriction of $f_{1}^{+}$to the boundary component $\partial D_{i} \subset \Sigma_{1}^{+}$agrees with the restriction of $f_{2}^{+}$onto $\partial D_{j} \subset \Sigma_{2}^{+}$. Assume that the boundary components $\partial D_{i}$ and $\partial D_{j}$ carry opposite orientations. Then we can sew the $M$-maps $\left(\Sigma_{k}^{+}, f_{k}^{+}\right)$along their boundaries $\partial D_{i_{k}}$ for $k=1,2$ and obtain a new one.

Let $C_{T_{1}} \times_{i j} C_{T_{2}}$ denote the space of pairs described above. This space is in fact a fiber product in the following way.

Recall that the space of algebraic loops is the space of morphisms $f: \mathbb{P}_{\mathbb{R}}^{1} \rightarrow \mathbb{P}_{\mathbb{R}}^{1}$. The components $C_{T_{k}}$ of moduli spaces $R_{g_{k}}\left(\mathbb{P}^{1}, d_{k}\right), k=1,2$, admit evaluation maps to $L^{\text {alg }}\left(\mathbb{P}_{\mathbb{R}}^{1}\right)$,

$$
e v_{i_{k}}: C_{T_{k}} \rightarrow L^{\text {alg }}\left(\mathbb{P}_{\mathbb{R}}^{1}\right),\left.\quad\left(\Sigma_{k}^{+}, f_{k}^{+}\right) \mapsto f_{k}^{+}\right|_{\partial D_{i_{k}}} .
$$

Then the space described above is the fibered product

$$
C_{T_{1}} \times_{L^{a l g}\left(\mathbb{P}_{\mathbb{R}}^{1}\right)} C_{T_{2}} .
$$

The sewing operation described above provides us with a morphism

$$
C_{T_{1}} \times_{i j} C_{T_{2}} \rightarrow C_{T_{1} \circ T_{2}} \text {. }
$$

\subsection{The trace}

Let $\left(\Sigma^{+}, f^{+}\right)$be in $C_{T} \subset R_{g}\left(\mathbb{P}^{1}, d\right)$ and let $S$ be the closed string which is the common boundary of a pair of weighted pants $P_{1}, P_{2}$ in $\Sigma^{+}$. Let $\left\{\partial D_{1}, \ldots, \partial D_{l}, S\right\}$ and $\left\{\partial D_{l+1}, \ldots, \partial D_{k}, S\right\}$ be the sets of boundaries of $P_{1}$ and $P_{2}$, respectively. Let the $w_{i}$ be the weights of $\partial D_{i}$ and $w_{s}$ be the weight of $S$. Let $T_{1}$ and $T_{2}$ be the weighted tangles of $P_{1}$ and $P_{2}$, respectively.

We first note that the deformations of such a pair of weighted pants and their maps to $\mathbb{P}^{1}$ are given by the fiber product

$$
C_{T_{1}} \times{ }_{s} C_{T_{2}}
$$

which is determined by the evaluation map $\left.\left(P_{i}, f_{i}\right) \mapsto f\right|_{S}$. As we have already seen above, there is a morphism of this product into the space $C_{T_{1} \circ_{s} T_{2}}$. This map in fact removes the string $S$ in $\Sigma^{+}$, i.e., it provides the trace operator in geometric terms in the above setting. This map is given by the gluing of a pair of weighted pants $P_{1}$ and $P_{2}$ as in [14]. In particular, if the closed string bounds a disc (rather than a more complicated weighted pant), then it plays the role of trace, and it is given as in (3.4).

The procedure of eliminating ovals in planar algebras, described in section 3.1.4, corresponds in algebro-geometric terms to the combinatorial data of gardens described in [14] consisting of planar chord diagrams decorated by rooted trees.

\subsection{The involution}

We can define an involution $*$ by replacing the complex structure $J$ of $\Sigma^{+}$by $-J$. This is equivalent to replacing $\Sigma^{+}$with the other half $\sigma\left(\Sigma^{+}\right)$of $\Sigma_{\mathbb{C}}$. This operation reverses the orientations of the strings and checkerboard shadings of the corresponding planar tangle. 


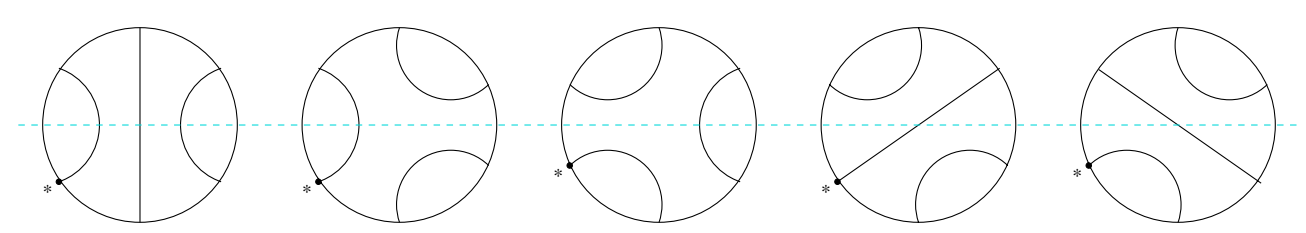

Figure 6. The bases of $T L_{3}$.
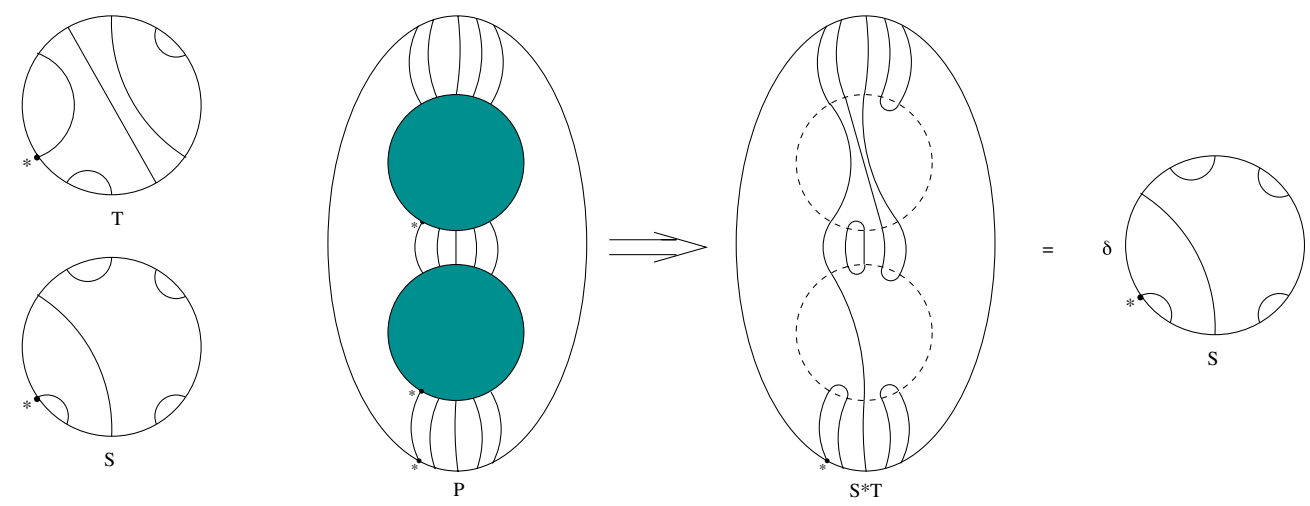

Figure 7. The product of tangles $S$ and $T$.

\subsection{Planar algebras and the moduli space of real maps $R_{g}\left(\mathbb{P}^{1}, d\right)$}

The collection of connected components of $R_{g}\left(\mathbb{P}^{1}, d\right)$ provides a topological operad $\mathcal{P}_{\mathbb{P}^{1}}$. The following statement then follows directly from the discussion of the previous subsections.

Theorem 4.4. The operad of (weighted) planar tangles is the topological operad $\mathcal{P}_{\mathbb{P}^{1}}$.

\subsection{An example: Temperley-Lieb algebras}

Consider the moduli space $R_{0}\left(\mathbb{P}^{1}, d\right)$ of $M$-maps of genus zero. Such $M$-maps have $2 d-2$ critical points. Then, the tangles that distinguish the components $C_{T}$ of $R_{0}\left(\mathbb{P}^{1}, d\right)$ are the planar tangles on a disc with $2 k$ points on their boundaries where $0 \leqslant 2 k \leqslant 2 d-2$. In other words, these tangles connect the first $k$ points starting at the marked critical point in $\Sigma^{+}$to the second $k$ points without having any crossings. These are in fact the tangles that generate the Temperley-Lieb algebras (modulo the trace). For instance, if we consider the case with $d=4$, we obtain the tangles that generate $T L_{1}, T L_{2}$ and $T L_{3}$; see figure 6 for the bases of $T L_{3}$.

The product of two such tangles is obtained by sewing their boundaries with a tangle of $M$-maps of genus 2. Figure 7 illustrates an example of a such a composition of tangles.

\section{Algebras over planar operad}

In this section, we discuss two possible geometric constructions of algebras over the planar operad $\mathcal{P}$. The first one follows closely the approach of Cohen and Godin's in constructing string topology operations [4]. The second one is in the spirit of Gromov-Witten theory [10], in the real algebraic geometry setting (see for instance [3]). 


\subsection{Planar algebras in string topology}

As we have already observed in section 4.3 , the moduli space $R_{g}\left(\mathbb{P}^{1}, d\right)$ admits evaluation maps

$$
e v_{i}: R_{g}\left(\mathbb{P}^{1}, d\right) \rightarrow L^{a l g}\left(\mathbb{P}_{\mathbb{R}}^{1}\right), \quad i=1, \ldots, g+1
$$

into the space of algebraic loops. Note that, like the usual topological loop space $L S^{1}$, the algebraic loop space $L^{\text {alg }}\left(\mathbb{P}_{\mathbb{R}}^{1}\right)$ has infinitely many connected components, each determined by the degree of the loops. However, in contrast to the topological case, the components of $L^{a l g}\left(\mathbb{P}_{R}^{1}\right)$ are finite dimensional and are not contractible, and are not compact. Therefore, they have nontrivial cohomology classes in the cohomology with compact support $\mathbb{H}^{*}=H_{c}^{*}\left(L^{\text {alg }}\left(\mathbb{P}_{\mathbb{R}}^{1}\right)\right)$.

By using these evaluations, we can pull back classes from $\mathbb{H}^{*}$ to the components $C_{T} \subset R_{g}\left(\mathbb{P}^{1}, d\right)$. Let $\gamma_{1}, \ldots, \gamma_{g+1} \in \mathbb{H}^{*}$. Then, we define topological correlators as

$$
\left\langle\gamma_{1}, \ldots, \gamma_{g+1}\right\rangle_{T}:=\int_{\left[C_{T}\right]} e v_{1}^{*}\left(\gamma_{1}\right) \wedge \cdots \wedge e v_{g+1}^{*}\left(\gamma_{g+1}\right) .
$$

Here the classes $e v_{1}^{*}\left(\gamma_{1}\right) \wedge \cdots \wedge e v_{g+1}^{*}\left(\gamma_{g+1}\right)$ are in the cohomology with compact support $H_{c}^{*}\left(C_{T}\right)$ since the components $C_{T} \subset R_{g}\left(\mathbb{P}^{1}, d\right)$ corresponding to the tangles $T$ are not compact due to the genericity condition of $M$-maps. Moreover, due to the stacky nature of the moduli space of $M$-maps, we pair the pulled back classes with the virtual fundamental class of $C_{T}$ (under the condition that dimensions match, otherwise, we simply set it to zero).

Let $\left\{e_{a}\right\}$ be a basis for $\mathbb{H}^{*}$ and let $g^{a b}$ be a non-degenerate pairing. We use the invariants in (5.1) of the loop space $L^{\text {alg }}\left(\mathbb{P}_{\mathbb{R}}^{1}\right)$ to give a representation of the operad $\mathcal{P}$ of planar tangles in the following way. The image of $T$ under the morphims $Z: \mathcal{P} \rightarrow \operatorname{Hom}\left(\left(\mathbb{H}^{*}\right)^{\otimes g}, \mathbb{H}^{*}\right)$ is

$$
T \mapsto Z(T):=\left(\gamma_{1} \otimes \cdots \otimes \gamma_{g} \mapsto \sum_{a, b}\left\langle\gamma_{1}, \ldots, \gamma_{g}, e_{a}\right\rangle_{T} g^{a b} e_{b}\right) .
$$

The composition of these higher products is evident from the definitions of these products in (5.2) and from the compositions of tangles described in section 4.3.

In this construction, the trace can be given by using the procedure described in section 4.4. Let $T$ be a planar tangle and let $S$ be closed in $T$. In such a case, we think of $T$ as a composition of two tangles $T_{1}$ and $T_{2}$ as in section 4.4. Then, we obtain this composition as in equation (3.2). In particular, if the closed string bounds a disc (rather than a more complicated weighted pant), then it plays the role of trace, and it is given as in (3.4). The planar tangles $T_{0, \emptyset, \mathcal{L}, b / w}$ in figure 4 identify the components of $R_{0}\left(\mathbb{P}^{1}, 2\right)$ (along with theta graphs). In this case, the recipe in (5.1) i.e., pulling back the fundamental class and integrating, gives us the degree of the evaluation map. In this particular case, we can immediately obtain that the degree is 1 since the evaluation map is an isomorphism between $C_{T_{0, \forall, Q, b / w}}$ and the component of $L^{\text {alg }}\left(\mathbb{P}_{\mathbb{R}}^{1}\right)$ parameterizing degree 2 maps. Therefore, we set $\delta_{1}=\delta_{2}=1$.

\subsection{Planar algebras via Gromov-Witten theory}

Another way to obtain a one-dimensional representation of $\mathcal{P}$ is to use ideas from GromovWitten theory of $\mathbb{P}^{1}$. In this case, we use a different evaluation map that maps $\left(\Sigma^{+}, f^{+}\right) \in C_{T}$ to its critical values in $\mathbb{P}^{1}$. The image of this evaluation map is the product of the space $\operatorname{Conf}\left(\mathbb{P}_{\mathbb{C}}^{1}, k\right)$ of (unordered) point configurations in $\mathbb{P}_{\mathbb{C}}^{1}$ (which contains the images of conjugate pairs critical points), the space $\operatorname{Conf}\left(\mathbb{P}_{\mathbb{R}}^{1}, l\right)$ of (unordered) point configurations in $\mathbb{P}_{\mathbb{R}}^{1}$ (which contains the images of real critical points except those marked at each boundary components) 
and the space $\widetilde{\operatorname{Conf}}\left(\mathbb{P}_{\mathbb{R}}^{1}, m\right)$ of ordered point configurations in $\mathbb{P}_{\mathbb{R}}^{1}$ (which contains the images of real critical points that are marked at each boundary components). The total number of critical points is $2 k+l+m$, and must be equal to $2(d+g-1)$.

Let $\gamma_{1}, \ldots, \gamma_{m} \in H^{1}\left(S^{1}\right)$, and $\left[\operatorname{Conf}\left(\mathbb{P}_{\mathbb{C}}^{1}, k\right)\right]$ and $\left[\operatorname{Conf}\left(\mathbb{P}_{\mathbb{R}}^{1}, l\right)\right]$ be the (relative) fundamental classes of the respective configuration spaces. We then define correlators by setting

$\left\langle\left\langle\gamma_{1}, \ldots, \gamma_{m}\right\rangle\right\rangle_{T}:=\int_{\left[C_{T}\right]} e v_{1}^{*}\left(\gamma_{1}\right) \wedge \cdots \wedge e v_{m}^{*}\left(\gamma_{m}\right) \wedge\left[\operatorname{Conf}\left(\mathbb{P}_{\mathbb{C}}^{1}, k\right)\right] \wedge\left[\operatorname{Conf}\left(\mathbb{P}_{\mathbb{R}}^{1}, l\right)\right]$.

The pulled-back classes again take values in the cohomology with compact support $H_{c}^{*}\left(C_{T}\right)$ of the component $C_{T} \subset R_{g}\left(\mathbb{P}^{1}, d\right)$ corresponding to the tangle $T$. Then, by using the same idea as in (5.2), we define the higher products corresponding to the tangles $T$ by setting

$$
T \mapsto Z(T):=\left(\gamma_{1} \otimes \cdots \otimes \gamma_{m} \mapsto\left\langle\left\langle\gamma_{1}, \ldots, \gamma_{m}\right\rangle\right\rangle_{T}\right) .
$$

The composition of these higher products and the trace operators are evident and are given as in the previous case described in section 5.1

Remark 5.1. Gromov-Witten invariants of $\mathbb{P}^{1}$ and the Hurwitz number are closely related. The relations for the complex case can be found in [16] and references there in. One expects to find a similar line of connection in the real case. On the other hand, in [14], the authors give a combinatorial formula for real Hurwitz numbers based on counting the planar tangles. Such a connection would provide a direct link between planar algebras and real Gromov-Witten theory.

Remark 5.2. Both situations discussed above, based on string topology or on GromovWitten theory, can be further enriched by using the tautological classes of the moduli space $R_{g}\left(\mathbb{P}^{1}, d\right)$. This might lead to additional interesting examples.

\section{Acknowledgments}

The first author would like to thank Vaughan Jones for useful discussions. The first author is partially supported by NWO. The second author is partially supported by NSF grants DMS0651925 and DMS-0901221. Part of this work was carried out during the authors' stay at the Max Planck Institute for Mathematics, which we thank for the hospitality and for support. The first author also thanks to Feza Gürsey Institute for the hospitality.

\section{References}

[1] Alling N L and Greenleaf N 1971 Foundations of the Theory of Klein Surfaces (Lecture Notes in Mathematics vol 219) (Berlin: Springer) ix+117pp

[2] Bisch D 2002 Subfactors and planar algebras Proc. Intl Congress of Mathematicians (Beijing, 2002) vol II pp 775-85

[3] Ceyhan Ö 2010 Towards quantum cohomology of real varieties in Arithmetic and Geometry Around Quantization volume, Prog. Math. 279 65-99

[4] Cohen R and Godin V 2004 A polarized view of string topology Topology, Geometry and Quantum Field Theory (London Math. Soc. Lecture Note Ser. 308) (Cambridge: Cambridge University Press) pp 127-54

[5] Guionnet A, Jones V F R and Shlyakhtenko D 2007 Random matrices, free probability, planar algebras and subfactors arXiv:0712.2904

[6] Jones V F R 1999 Planar algebras: I arXiv:math.QA/9909027

[7] Katz S and Liu C C M 2001 Enumerative geometry of stable maps with Lagrangian boundary conditions and multiple covers of the disc Adv. Theor. Math. Phys. 5 1-49 
[8] Kodiyalam V and Sunder V S 2004 A complete set of numerical invariants for a subfactor J. Funct. Anal. 212 1-27

[9] Kodiyalam V and Sunder V S 2009 From subfactor planar algebras to subfactors Int. J. Math. 20 1207-31

[10] Kontsevich M and Manin Y U 1994 Gromov-Witten classes, quantum cohomology, and enumerative geometry Commun. Math. Phys. 164 525-62

[11] Liu C C M 2002 Moduli of $J$-holomorphic curves with Lagrangian boundary conditions and open GromovWitten invariants for an $S^{1}$-equivariant pair arXiv:math/0210257

[12] May J P 1997 Definitions: operad, algebras and modules Contemp. Math. 202 1-7

[13] Natanzon S and Shadrin S 2003 Topological classification of real meromorphic functions in general position on separating curves Dokl. Akad. Nauk 388 449-51

[14] Natanzon S, Shapiro B and Vainshtein A 2002 Topological classification of generic real rational functions $J$. Knot Theor. Ramific. 11 1063-75

[15] Natanzon S 1993 Topology of 2-dimensional coverings and meromorphic functions on complex and real algebraic curves Sel. Math. Sov. 12 251-91

[16] Okounkov A and Pandharipande R 2009 Gromov-Witten theory, Hurwitz numbers, and matrix models Algebraic Geometry-Seattle 2005: Proc. Symp. Pure Math. vol 80, part 1 (Providence, RI: American Mathematical Society) pp 325-414

[17] Popa S 1995 An axiomatization of the lattice of higher relative commutants of a subfactor Invent. Math. 120 427-45

[18] Seppälä M 1991 Moduli spaces of stable real algebraic curves Ann. Sci. École Norm. Sup. 24 519-44 